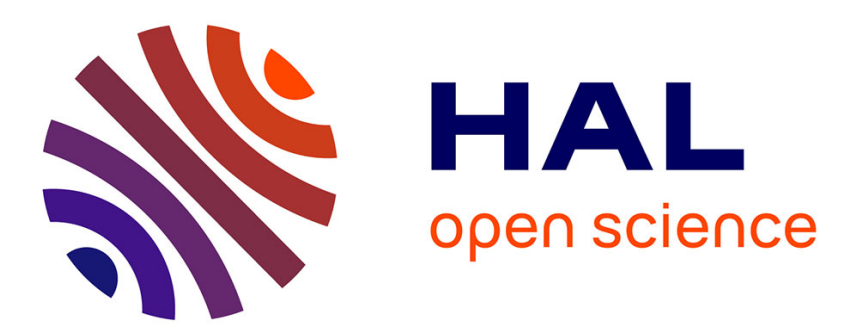

\title{
Comparison of Residual and Hierarchical Finite Element Error Estimators in Eddy Current Problems
}

Zuqi Tang, Patrick Dular, Yvonnick Le Menach, Emmanuel Creusé, Francis Piriou

\section{- To cite this version:}

Zuqi Tang, Patrick Dular, Yvonnick Le Menach, Emmanuel Creusé, Francis Piriou. Comparison of Residual and Hierarchical Finite Element Error Estimators in Eddy Current Problems. COMPUMAG 2013, Jul 2013, Budapest, Hungary. 10.1109/TMAG.2013.2285254 . hal-01947704

\author{
HAL Id: hal-01947704 \\ https://hal.science/hal-01947704
}

Submitted on 7 Dec 2018

HAL is a multi-disciplinary open access archive for the deposit and dissemination of scientific research documents, whether they are published or not. The documents may come from teaching and research institutions in France or abroad, or from public or private research centers.
L'archive ouverte pluridisciplinaire HAL, est destinée au dépôt et à la diffusion de documents scientifiques de niveau recherche, publiés ou non, émanant des établissements d'enseignement et de recherche français ou étrangers, des laboratoires publics ou privés. 


\title{
Comparison of Residual and Hierarchical Finite Element Error Estimators in Eddy Current Problems
}

\author{
Zuqi Tang ${ }^{1,4}$, Patrick Dular ${ }^{2,3}$, Yvonnick Le Ménach ${ }^{4}$, Emmanuel Creusé $^{1}$, Francis Piriou ${ }^{4}$ \\ ${ }^{1}$ Université Lille 1, LPP UMR 8524 and INRIA Lille Nord Europe, Villeneuve d'Ascq, F-59655 France \\ ${ }^{2}$ University of Liège, Dept. of Electrical Engineering and Computer Science, ACE, B-4000 Liège, Belgium \\ ${ }^{3}$ F.R.S.-FNRS, Fonds de la Recherche Scientifique, Belgium \\ ${ }^{4}$ Université Lille1, L2EP, Villeneuve d'Ascq, F-59655 France.
}

\begin{abstract}
The finite element computation of eddy current problems gives numerical error. This error cannot be calculated, but can only be estimated. Among all error estimators already developed, it is proposed to compare two proven estimators called residual and hierarchical error estimators.
\end{abstract}

\section{INTRODUCTION}

To evaluate the quality of numerical computation of low frequency electromagnetic finite element (FE) problems, a lot of error estimators have been developed. Some of them are based on energy minimization. Others, called equilibrated estimator, are based on the verification of the constitutive relations. More recently the residual a posteriori error estimator (REE) [1-4] and the hierarchical error estimator (HEE) have been proposed [5]. In the case of the REE, all the terms weakly verified are evaluated to obtain an estimation of the error [4]. On the other hand, with the HEE, the error estimation is given by using hierarchical higher order (HO) test functions (TFs) [6] in the FE formulation, either to evaluate some residues or to obtain HO solutions [5].

In this paper, we propose to apply the REE to FEs up to second order and to compare the obtained results with the HEE on the same mesh.

\section{ERROR ESTIMATORS}

\section{A. Weak finite element magnetodynamic formulation}

The weak magnetic vector potential $\boldsymbol{a}$-formulation of the magnetodynamic problem is obtained from the weak form of the Ampère equation, i.e. [5],

$$
\left(\boldsymbol{h}, \operatorname{curl} \boldsymbol{a}^{\prime}\right)_{\Omega}-\left(\boldsymbol{j}, \boldsymbol{a}^{\prime}\right)_{\Omega}+<\boldsymbol{n} \times \boldsymbol{h}, \boldsymbol{a}^{\prime}>_{\Gamma}=0, \forall \boldsymbol{a}^{\prime} \in F^{1}(\Omega),
$$

where $F^{1}(\Omega)$ is a curl-conform function space defined on $\Omega$; $(\cdot, \cdot)_{\Omega}$ and $<\cdot, \cdot>_{\Gamma}$ denote a volume integral in $\Omega$ and a surface integral on $\Gamma$, respectively, of the product of their field arguments; $\boldsymbol{n}$ is the unit normal on $\Gamma$ exterior to $\Omega$. Magnetic field $\boldsymbol{h}$ and electric current density $\boldsymbol{j}$ are related to magnetic flux density $\boldsymbol{b}$ and electric field $\boldsymbol{e}$ (in $\Omega_{\mathrm{c}} \subset \Omega$, with $\Omega=\Omega_{c} \cup \Omega_{c}{ }^{C}$ ), respectively, through the material relations $\boldsymbol{h}=\mu^{-1} \boldsymbol{b}$ and $\boldsymbol{j}=\boldsymbol{j}_{\mathrm{s}}+\sigma \boldsymbol{e}$, with $\mu$ the magnetic permeability, $\sigma$ the electric conductivity and $\boldsymbol{j}_{\mathrm{S}}$ the source current density in stranded inductors in $\Omega_{\mathrm{S}} \subset \Omega_{c}{ }^{C}$. With the magnetic vector potential $\boldsymbol{a}$ defined via $\boldsymbol{b}=\operatorname{curl} \boldsymbol{a}$ and $\boldsymbol{e}=-\partial_{t} \boldsymbol{a}-\operatorname{grad} v\left(\partial_{t}\right.$ is $j \omega$ with $\omega$ the angular frequency in the frequency domain), weak formulation (1) gives the $\boldsymbol{a}-\boldsymbol{v}$ formulation (or $\boldsymbol{A}-\varphi$ formulation with notations of $[4,5])$, i.e.

$$
\begin{array}{r}
\left(\mu^{-1} \operatorname{curl} \boldsymbol{a}, \operatorname{curl} \boldsymbol{a}^{\prime}\right)_{\Omega}+\left(\sigma \partial_{t} \boldsymbol{a}, \boldsymbol{a}^{\prime}\right)_{\Omega_{c}}+\left(\sigma \operatorname{grad} v, \boldsymbol{a}^{\prime}\right)_{\Omega_{c}} \\
-\left(\boldsymbol{j}_{s}, \boldsymbol{a}^{\prime}\right)_{\Omega_{s}}+<\boldsymbol{n} \times \boldsymbol{h}, \boldsymbol{a}^{\prime}>_{\Gamma}=0, \forall \boldsymbol{a}^{\prime} \in F^{1}(\Omega),
\end{array}
$$

with $F^{1}(\Omega)$ gauged in $\Omega_{c}{ }^{C}$, and containing the basis functions for $\boldsymbol{a}$ as well as for the test function $\boldsymbol{a}^{\prime}$ (at the discrete level, this space is defined by edge FEs; the gauge is based on the tree-co-tree technique); note that $F^{1}(\Omega)$ can be non-gauged if a proper resolution scheme is used [4]. Once a solution is obtained in a particular discrete function space with (2), it can be used $a$ posteriori to estimate the associated error.

\section{B. Residual error estimator (REE)}

Generally, the discretization error committed by the FE method can be resumed by the error on the energy. In the case of the $\boldsymbol{a}-v$ formulation, the local error on $\boldsymbol{a}$ and $v$ can be written $\boldsymbol{e}_{a}=\boldsymbol{a}-\boldsymbol{a}_{h}$ and $\boldsymbol{e}_{v}=v-v_{h}$, with $\boldsymbol{a}$ and $v$ the exact solutions of the problem and $\boldsymbol{a}_{h}$ and $v_{h}$ the numerical ones. From these local errors, the global error norm can be written, involving error terms on magnetic energy and Joule losses, i.e.

$$
\varepsilon^{2}=\int_{\Omega} \mu^{-1}\left|\operatorname{curl} \boldsymbol{e}_{a}\right|^{2}+\int_{\Omega_{c}} \frac{\sigma}{\omega}\left|j \omega \boldsymbol{e}_{a}+\operatorname{grad} e_{v}\right|^{2} .
$$

Carrying out an ad-hoc Helmholtz decomposition of (1) and using suitable interpolation operators and Cauchy-Schwarz inequality, the efficiency and the reliability of REE can be obtained [4]. For the $\boldsymbol{a}-\boldsymbol{v}$ formulation, the REE $\eta_{\omega}$ on FE $\omega \subset \Omega$ quantifies all the terms weakly verified in (2) via

$$
\eta_{\omega}^{2}=\eta_{\omega ; 1}^{2}+\eta_{\omega ; 2}^{2}+\eta_{\omega ; 3}^{2}+\sum_{\gamma \subset \partial \omega}\left(\eta_{\gamma ; 1}^{2}+\eta_{\gamma ; 2}^{2}\right)
$$

with

$$
\begin{aligned}
& \eta_{\omega ; 1}=h_{\omega}\left\|\pi_{h} \boldsymbol{j}_{S}-\operatorname{curl}\left(\mu^{-1} \operatorname{curl} \boldsymbol{a}\right)-\sigma(j \omega \boldsymbol{a}+\operatorname{grad} v)\right\|_{\omega}, \\
& \eta_{\omega ; 2}=h_{\omega}\|\operatorname{div}(\sigma(j \omega \boldsymbol{a}+\operatorname{grad} v))\|_{\omega}, \\
& \eta_{\omega ; 3}=h_{\omega}\left\|\boldsymbol{j}_{S}-\pi_{h} \boldsymbol{j}_{S}\right\|_{\omega}, \\
& \eta_{\gamma ; 1}=h_{\gamma}^{1 / 2}\left\|\left[\boldsymbol{n} \times \mu^{-1} \operatorname{curl} \boldsymbol{a}\right]_{\gamma}\right\|_{\gamma^{\prime}}, \\
& \eta_{\gamma ; 2}=h_{\gamma}^{1 / 2}\left\|[\sigma(j \omega \boldsymbol{a}+\operatorname{grad} v) \cdot \boldsymbol{n}]_{\gamma}\right\|_{\gamma^{\prime}} .
\end{aligned}
$$

Operator $\pi_{\mathrm{h}}$ represents the projection operator from $\mathrm{H}(\operatorname{div}, \Omega)$ in a discrete approximation space. Coefficients $h_{\omega}$ and $h_{\gamma}$ stand for the diameter of the smallest sphere containing the element $\omega$ and the diameter of the smallest circle containing the facet $\gamma$, respectively. All these terms must be evaluated on 
each FE. Terms (5a-b) quantify the error of the equation to solve. Term (5c) corresponds to the discretization error of the source term. Finally, terms (5d-e) evaluate the jumps (discontinuities) of the tangential component of $\boldsymbol{h}$ and the normal component of $\mathbf{j}$ through the element facets.

\section{Hierarchical error estimator (HEE)}

Once weak form (2) has been solved, it is evaluated with the corresponding solution with $\mathrm{HO}$ basis functions (BFs) $\boldsymbol{a}_{p}{ }^{\prime} \in F_{p}{ }^{1}(\Omega)$ as TFs. This gives a left hand side of (2) not equal to zero anymore but defining a residue $R_{p}$ that can serve as an error estimator, called HEE [5], i.e.

$$
\begin{gathered}
R_{p}=\left(\mu^{-1} \operatorname{curl} \boldsymbol{a}, \operatorname{curl} \boldsymbol{a}_{p}{ }^{\prime}\right)_{\Omega}+\left(\sigma \partial_{t} \boldsymbol{a}, \boldsymbol{a}_{p}{ }^{\prime}\right)_{\Omega_{c}}+\left(\sigma \operatorname{grad} v, \boldsymbol{a}_{p}{ }^{\prime}\right)_{\Omega_{c}} \\
-\left(\boldsymbol{j}_{s}, \boldsymbol{a}_{p}{ }^{\prime}\right)_{\Omega_{s}}+<\boldsymbol{n} \times \boldsymbol{h}, \boldsymbol{a}_{p}{ }^{\prime}>_{\Gamma}, \boldsymbol{a}_{p}{ }^{\prime} \in F_{p}^{1}(\Omega)
\end{gathered}
$$

Hierarchical HO-BFs can be associated with edges, facets and volumes of finite elements (FEs) [6]. For an edge or a facet HO BF $\boldsymbol{a}_{p}{ }^{\prime}$, respectively in 2-D and 3-D, shared by two FEs $\omega_{a}$ and $\omega_{b}$, with interface $\gamma_{a b}$, residue (6) has a contribution only from these two FEs. Restricting the form (6) to $\omega_{a}$ and $\omega_{b}$ separately, with $\boldsymbol{a}_{p}{ }^{\prime}$ equal to zero on all the boundaries except $\gamma_{a b}$, integrating their first volume integral term by parts, and adding the resulting forms, one gets

$$
R_{p}=\left(\operatorname{curl} \boldsymbol{h}-\boldsymbol{j}, \boldsymbol{a}_{p}{ }^{\prime}\right)_{\omega_{a}+\omega_{b}}-<\boldsymbol{n}_{a b} \times\left(\left.\boldsymbol{h}\right|_{\omega_{a}}-\left.\boldsymbol{h}\right|_{\omega_{b}}, \boldsymbol{a}_{p}{ }^{\prime}\right)>_{\gamma_{a b}},
$$

with $\boldsymbol{n}_{a b}=\left.\boldsymbol{n}\right|_{\partial \omega_{a}}=-\left.\boldsymbol{n}\right|_{\partial \omega_{b}}$ the unit normal to $\gamma_{a b}$ exterior to $\omega_{a}$. Residue $R_{p}$, calculated via (6), is therefore a union of two errors, pointed out by (7): an error on the equation to solve, via the volume integral term on $\omega_{a}+\omega_{b}$, and an error on the tangential continuity of $\boldsymbol{h}$, via the surface integral term on $\gamma_{a b}$.

For an HO-BF $\boldsymbol{a}_{p}{ }^{\prime}$ associated with the volume of a FE $\omega_{a}$, thus with a zero trace on $\partial \omega_{a}$, the residue given by (6) is reduced to

$$
R_{p}=\left(\operatorname{curl} \boldsymbol{h}-\boldsymbol{j}, \boldsymbol{a}_{p}{ }^{\prime}\right)_{\omega_{a}} .
$$

Using TFs $\boldsymbol{a}^{\prime}=\operatorname{grad} v^{\prime}$ in (2) (which is implicitly the case with edge FEs for $\boldsymbol{a}^{\prime}$, that contains the gradient of nodal FEs), weak form (2) becomes the weak form of $\operatorname{div} \boldsymbol{j}=0$, i.e.

$$
\left(\sigma \partial_{t} \boldsymbol{a}, \operatorname{grad} v^{\prime}\right)_{\Omega_{c}}+\left(\sigma \operatorname{grad} v, \operatorname{grad} v^{\prime}\right)_{\Omega_{c}}-<n \cdot j, v^{\prime}>_{\Gamma_{s}}=0,
$$

with $\Gamma_{s}$ some possible cross sections of conductors in $\Omega_{c}$. A residue $R_{v, p}$ can be also defined for (9) when using HO TFs $v_{p}{ }^{\prime}$ for $v^{\prime}$, being also equal to

$$
R_{v, p}=\left(\operatorname{div} \boldsymbol{j}, v_{p}{ }^{\prime}\right)_{\omega_{a}+\omega_{b}}-<\boldsymbol{n}_{a b} \cdot\left(\left.\boldsymbol{j}\right|_{\omega_{a}}-\left.\boldsymbol{j}\right|_{\omega_{b}}, v_{p}{ }^{\prime}\right)>_{\gamma_{a b}} .
$$

Residue $R_{v, p}$ points out an error on $\operatorname{div} \boldsymbol{j}=0$ on $\omega_{a}+\omega_{b}$ and an error on the normal continuity of $\boldsymbol{j}$ on $\gamma_{a b}$.

A useful additional step can consist in using $\mathrm{R}_{p}$ as a source (right hand side) for a local FE problem (limited to the FE support of each TF), calculating the HO correction $\boldsymbol{a}_{p}$ to be given to solution $\boldsymbol{a}$ for satisfying the $\mathrm{HO}$ weak formulation [5], i.e.

$$
\left(\mu^{-1} \operatorname{curl} \boldsymbol{a}_{p}, \operatorname{curl} \boldsymbol{a}_{p}{ }^{\prime}\right)_{\Omega}+\left(\sigma \partial_{t} \boldsymbol{a}_{p}, \boldsymbol{a}_{p}{ }^{\prime}\right)_{\Omega_{c}}+\left(\sigma \operatorname{grad} v_{p}, \boldsymbol{a}_{p}{ }^{\prime}\right)_{\Omega_{c}}=-R_{p} .
$$

This allows an error estimation directly relative to the scale of the solution, whereas $\mathrm{R}_{p}$ is only relative to the others residues for the same mesh (as for the REE; the direct use of $\mathrm{R}_{p}$ can define another kind of REE).

\section{COMPARISON OF ERROR ESTIMATORS}

REE and HEE show some equivalences and differences. Equivalences can be found in all the volume and surface integral contributions involved in the estimators, where both weakly defined equations and interface conditions are evaluated in a way to express their related contributions to the errors. A difference appears regarding the weighting of the contributions, with coefficients defined as smallest diameters for the REE while such coefficients are implicitly defined via integrations in each FE with the HEE. This gives differences, in particular in anisotropic meshes.

Local contributions to the errors are related to each single FE with the REE and with groups of FEs (sharing edges or facets) or single FE (for volume TFs) with the HEE. In both estimators, the contributions are only relative to other similar contributions (which can be useful for mesh adaption), and are thus not expressed at the scale of the solution. However, the HEE can be extended to give a solution-relative error by calculating $\mathrm{HO}$ corrections.

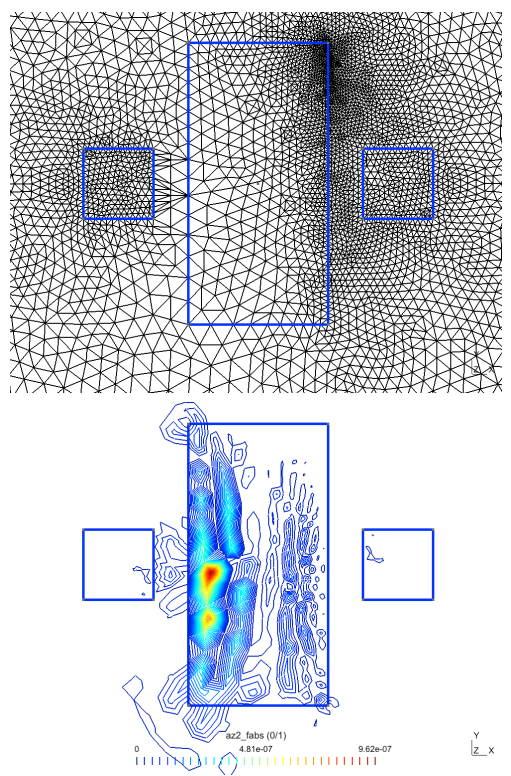

Fig. 1. Example of distribution of local error estimation in an eddy current problem. The highest error is located in the skin depth with a too coarse mesh.

\section{REFERENCES}

[1] I. Babuska and W. C. Rheinboldt, "A posteriori error estimates for the finite element method", International Journal for Numerical Methods in Engineering, vol 12, pp. 1597-1615, 1978.

[2] R. Beck, R. Hiptmair, R.H.W. Hoppe, and B. Wohlmuth, "Residual based a posteriori error estimators for eddy current computation", ESAIM: Mathematical Modelling and Numerical Analysis, 34(01):159$182,2000$.

[3] J. Chen, Z. Chen, T. Cui, and L.B. Zhang. "An adaptive finite element method forthe eddy current model with circuit/field couplings", SIAM Journal on Scientific Computing, 32(2) :1020, 2010.

[4] E. Creusé, S. Nicaise, Z. Tang, Y. Le Menach, N. Nemitz, and F. Piriou, "Residual-based a posteriori estimators for the $\mathrm{A} / \mathrm{Phi}$ magnetodynamic harmonic formulation of the Maxwell system", Mathematical Models and Methods in Applied Sciences, 22(5):30, 2012.

[5] P. Dular, "A posteriori error estimation of finite element solutions via the direct use of higher order hierarchal test functions", IEEE Trans. Magn., vol.45, no.3, pp.1360-1363, March 2009.

[6] J. P. Webb and B. Forghani, "Hierarchal scalar and vector tetrahedra", IEEE Trans. Magn., Vol.29, No.2, pp. 1495-1498, 1993. 